\title{
CHILDHOOD MORTALITY: STILL A GLOBAL PRIORITY
}

\author{
Hani K. Atrash MD, MPH*
}

\begin{abstract}
Mortality of children under-5 continues to be a global priority. In 2012, 6.6 million children under5 died worldwide; more than half of these deaths are due to diseases that are preventable and treatable through simple, affordable interventions. In response to the United Nations' Millennium Development Goal (MDGs) which called, through MDG4, to "reduceby two thirds the under-5 child mortality, between 1990 and 2015", global organizations and many countries set targets and developed specific strategies to reduce child mortality and monitor progress. As a result, the number of deaths in children under-5 worldwide declined from 12.4 million in 1990 to 6.6 in 2012 . Under-5 child mortality dropped in all regions of the world. However, two major challenges face the international community: The wide disparity in the risk of child death among countries, and the emerging role of neonatal death as a major component of child mortality. In order to continue the progress in reducing under-5 child mortality worldwide, current efforts must continue and new strategies need to be implemented to focus on preventing neonatal deaths as they start to represent a larger proportion of under-5 child deaths. In particular, further reduction in neonatal mortality will depend heavily on improving maternal health (MDG5). The world leaders continue to support the MDGs. In 2010, in a major push to accelerate progress on women's and children's health, a number of Heads of State and Government from developed and developing countries, along with the private sector, foundations, international organizations, civil society and research organizations, pledged over $\$ 40$ billion in resources over the next five years.
\end{abstract}

Key words: childhood mortality, child health, global health, millennium development goals

Millions of children die each year worldwide. Most of these deaths occur in developing countries and most can be prevented using currently recommended simple, affordable, and effective interventions. Significant progress has been made in preventing childhood deaths since the adoption of the Millennium Development Goals in $2000^{1}$. However, efforts need to continue and be intensified to achieve the MDG 4 (Reduce child mortality) and, with the changes in the leading causes of childhood mortality, new strategies need to be developed and adopted to achieve further progress.

Epidemiology: Because of the biologic and social vulnerability of children under 5 years of age and because childhood mortality rates decrease dramatically after the age of 5, "Childhood Death" is typically defined among the global public health community as death of persons younger than 5 years of age ${ }^{2}$. Under-5 childhood death (U5CD) rates are also commonly used as indicators to assess the health of a general population. The majority of the
U5CD occur in developing countries. For example, children in sub-Saharan Africa are about 15 times more likely to die before the age of five than children in developed regions ${ }^{3,4}$.

It is estimated that 6.6 million children under the age of five died in $2012^{3,4}$. More than half of these early child deaths are due to conditions that could be prevented or treated with access to simple, affordable interventions. The leading causes of death in under-five children are pneumonia, preterm birth complications, diarrhea, birth asphyxia and malaria. About one third of all child deaths are linked to malnutrition ${ }^{4-6}$.

An analysis of the estimated 8.8 million U5CDS worldwide concluded that $68 \%$ were caused by infectious diseases ( $18 \%$ caused by pneumonia, diarrhea $15 \%$, and malaria $8 \%$ ). (5) Malnutrition is the underlying contributing factor in over one third of all child deaths, making children more vulnerable to severe diseases ${ }^{6}$. In 2010 about 20 million children worldwide were estimated to suffer from severe

Director, Division of Healthy Start and Perinatal Services. Maternal and Child Health Bureau. Health Resources and Services Administration

Corresponding author: hatrash@hrsa.gov

Suggested citation: Atrash HK. Childhood mortality: still a global priority. Journal of Human Growth and Development. 2013; 23(3): 257-260.

Manuscript submitted Aug 01 2013, accepted for publication Sep 192013. 
acute malnutrition, leaving them more vulnerable to serious illness and early death. Most of these children can be successfully treated at home with ready-to-use therapeutic foods (RUTF).

Globally, in 2010, an estimated 171 million children below five years of age were stunted and 104 million were underweight. Malnourished children, particularly those with severe acute malnutrition, have a higher risk of death from common childhood illness such as diarrhea, pneumonia, and malaria. Globally, nearly one in six children under age five are underweight; one in four are stunted. Nutrition-related factors contribute to about $45 \%$ of deaths in children under five years of age ${ }^{7,8}$.

More than half of U5CDs are due to diseases that are preventable and treatable through simple, affordable interventions. Strengthening health systems to provide such interventions to all children will save many young lives. For example, vaccine preventable diseases account for $14 \%$ of the U5CDs and vaccines against tuberculosis, measles, polio, diphtheria, tetanus, pertussis, pneumonia due to Haemophilius influenzae type B and Streptococcus pneumoniae and diarrhea due to rotavirus, are available and can protect children from illness and death $^{8}$. Prevention and treatment of other illnesses causing death are also available. Diarrhea could be prevented through ensuring clean water, sanitation and hygiene. Simple approaches for treating diarrhea include oral rehydration therapy, increased fluids, home-made sugar/salt/water solutions, oral rehydration salts, continued feeding (breastfeeding), and selective use of antibiotics.

Similarly, prevention of pneumonia may be achieved through immunization (measles, pertussis), exclusive breastfeeding/appropriate complementary feeding, vitamin A and zinc supplementation, avoidance of indoor air pollution, and hand hygiene. Treatment includes prompt case management and the administration of antibiotics. Finally, prevention of malaria can be accomplished through mosquito control, indoor residual spraying, environmental measures (e.g. reduction of standing water), and insecticide treated nets. Malaria can be treated through prompt administration of antimalarial drugs ${ }^{6,8}$.

The Global Response: In September 2000, the world leaders came together at the United Nations Headquarters in New York to adopt the United Nations Millennium Declaration, and set out the eight Millennium Development Goals (MDGs) to be achieved by 2015 . The Goals range from cutting in half extreme poverty to halting the spread of HIV/ AIDS and providing universal primary education ${ }^{1}$.

One of the MDGs focuses on childhood mortality. MDG 4 calls for "Reducing child mortality" and sets a target to "Reduce by two thirds the underfive child mortality, between 1990 and 2015". Linked to this Goal and indirectly contributing to reducing U5CD, is MDG 5 which focuses on "Improving maternal health" and sets two targets by 2015:
1. "Reduce by three-quarters, between 1990 and 2015, the maternal mortality ratio", and

2. "Achieve, by 2015, universal access to reproductive health" ${ }^{1}$.

The MDGs raised awareness, identified child mortality as one of the eight priorities for global action, and called the world leaders to act to achieve the MDGs. In response, global organizations and many countries set targets and developed specific strategies to reduce child mortality and monitor progress. Since then, big gains have been made in child survival ${ }^{7,8}$.

Between the years 2000 and 2011, measles vaccines have averted over 10 million deaths. Measles vaccination rates have increased in most regions, particularly in sub-Saharan Africa and Southern Asia. Global coverage of the first-dose measles vaccine increased from 72 per cent to 84 per cent. An estimated 10.7 million deaths were averted from 2000 to 2011 due to immunizations against measles ${ }^{7}$.

The United Nations Children's Fund (UNICEF) 2013 Child Mortality Report indicates that the number of deaths in children under five worldwide declined from 12.4 million in 1990 to 6.6 in 2012 . The U5CD rate dropped $47 \%$ during the same period from 90 to 48 deaths per 1,000 births ${ }^{2,3}$.

The U5CD rate dropped in all regions of the world. Overall the U5CD rate decreased by $2.9 \%$; the annual reduction rate was higher in developed regions $(3.8 \%)$ than in developing region $(2.9 \%)$. The reduction rate was highest in Eastern Asia $(6.1 \%)$ and lowest in Oceania (1.4\%) and in SubSaharan Africa (2.7\%). Eastern Asia and Northern Africa have already exceeded the MDG 4 with a reduction of $74 \%$ and $69 \%$ respectively. Reductions in Latin America and the Caribbean (65\%) and Western Asia (62\%) indicate that these regions are on their way to achieving the MDG 4. However, SubSaharan Africa and Oceania have lagged behind and witnessed reductions of only $45 \%$ and $26 \%$ respectively ${ }^{3}$.

At the country level, most countries have seen significant reduction in child mortality between 1990 and 2012. Of the 61 high-mortality countries with at least 40 deaths per 1,000 live births in

2012, 25 have reduced their U5CD rate by at least half between 1990 and 2012. Seven of these countries have already achieved the MDG 4 and reduced their mortality rates by more than $66 \%$ (Bangladesh, Malawi, Nepal, Liberia, Tanzania, Timor-Leste, and Ethiopia) ${ }^{3}$.

There have also been significant improvements in maternal health (MDG5). The United nations estimates that between 1990 and 2011 , the maternal mortality rate has declined by $47 \%$ from an estimated 400 maternal deaths per 100,000 live births to an estimated rate of 200 . In developing regions, the proportion of deliveries attended by skilled personnel rose from $55 \%$ to $66 \%$, and coverage of prenatal care (at least one visit with 
a doctor, nurse or midwife during pregnancy) increased from $63 \%$ to $81 \%$. (7) Yet, the maternal mortality rate continues to be very high, nearly 50 million babies worldwide are delivered without skilled care, and a large proportion of women do not get any prenatal care?

Challenges to achieving the MDG4: Despite the enormous accomplishments summarized above, more rapid progress is needed to meet the 2015 target of a two thirds reduction in child deaths. In 2012, more than 18,000 children died every day, mostly from preventable diseases ${ }^{3}$.

Today, two major challenges face the international community:

1. The wide disparity in the risk of child death among countries, and

2. The emerging role of neonatal death as a major component of child mortality.

First, in 2012, the U5CD rates range from 6 per 1,000 births in developed countries to 53 in developing countries. $49 \%$ of child deaths occurred in five countries: India, Nigeria, Democratic Republic of the Congo, Pakistan, and China ${ }^{8}$. In 2012, most of the U5CDs occurred in the poorest regions and countries of the world, and in the most underprivileged areas within countries of SubSaharan Africa and Southern Asia where U5CD rates were 98 and 58 respectively. In 2012, these two regions accounted for $82 \%$ of the U5CDs worldwide (5.4 of the 6.6 million). In sub-Saharan Africa, 1 in 9 children die before age five; in Southern Asia, 1 in 16. Mortality rates in some countries of these regions are estimated to reach 200 deaths per 1,000 births; in other words, 1 of every 5 babies born dies before reaching the age of $5^{1}$. Within countries, child mortality is higher in rural areas, and among poorer and less educated families.

The second challenge is the increase in the share of neonatal deaths among U5CDs (deaths before the age of 29 days). Neonatal mortality rates are declining at a slower rate than the overall U5CD rates. From 1990 to 2012, the global neonatal mortality rate decreased by $37 \%$, from 33 to 21 deaths per 1,000 live births. This decline is much less than the overall $47 \%$ decline in the U5CD rate. This slower decline in neonatal mortality rate compared to the decline in the overall $U 5 C D$ rate resulted in an increase in the share of neonatal deaths among under-five deaths from $37 \%$ in 1990 to $44 \%$ in 2012 .

This share is also higher in areas with low U5CD rates and lower in areas with high U 5CD rates. Thus in the developed regions in 2012, the neonatal deaths as a share of U5CDs was $56 \%$, compared with $43 \%$ in developing regions overall but $26 \%$ in Sub-Saharan Africa and $41 \%$ in Latin America and the Caribbean ${ }^{3}$.

Furthermore, variation in neonatal mortality rates among countries is substantial; the neonatal mortality rates range from 4 deaths per 1,000 live births in developed countries to rates exceeding 30 in countries of Sub-Saharan Africa ${ }^{3}$. Approximately two-thirds of neonatal deaths occur in just 10 countries, with India accounting for more than a quarter and Nigeria for a tenth of the total number of neonatal deaths.

A larger component of efforts to reduce neonatal mortality falls on the shoulders of the maternal and child health community with renewed focus on improving maternal health and maternal care during pregnancy, labor and delivery, as well as a focus on neonatal health and neonatal care. Many of the children who die before 28 days of life die at home in the first week of life and many suffer from diseases and conditions that are readily preventable or treatable with proven, cost-effective interventions delivered during the pre- , intra- , and postpartum periods. Globally, over $80 \%$ of neonatal deaths are caused by complications of prematurity and low birth weight, neonatal infections, and asphyxia ${ }^{5,6,8}$. Interventions to prevent these deaths include prenatal screening and care, delivery by skilled birth attendants, promotion of early and exclusive breastfeeding, hygienic skin and umbilical cord care, keeping babies warm and dry, family recognition of signs of illnesses requiring prompt medical attention, and early assessment of newborns by trained health workers ${ }^{6,9}$. Scaling up low-cost solutions to address preterm birth could reduce these deaths by three quarters ${ }^{9}$.

What will it take to meet MDG4? Despite significant improvement in reducing U5CDs worldwide, meeting the MDG4 target by 2015 will require greater focus and a renewed commitment to reaching the most vulnerable children. Current efforts must continue and new strategies need to be implemented to focus on preventing neonatal deaths as they start to represent a larger proportion of U5CDs. In this regard, further progress in reducing U5CDs and achieving the MDDG4 will also depend on progress toward achieving the MDG5 (improving maternal health). The world leaders continue to support the MDGs and have adopted a global action plan in 2010 to invest in achieving the MDGs following the 2010 Summit on the Millennium Development Goals titled "Keeping the Promise - United to Achieve the Millennium Development Goals".

In particular, in a major push to accelerate progress on women's and children's health, a number of Heads of State and Government from developed and developing countries, along with the private sector, foundations, international organizations, civil society and research organizations, pledged over \$40 billion in resources over the next five years ${ }^{10}$.

This commitment by the international leadership provides optimism that further progress towards achieving the MDG4 can be attained. With the share of under-five deaths during the neonatal period rising in every region and almost all countries, neonatal health will need to be addressed more effectively. Systematic action by governments and partner organizations is needed to reach women and babies with effective care. Highly cost-effective interventions are feasible even at the community level, and most can be linked with preventive and curative initiatives for mothers and babies. 


\section{REFERENCES:}

1. United Nations - Millennium Development Goals. http: //www.un.org/millenniumgoals/ bkgd.shtml. Accessed November 20, 20139.

2. Wang $H$, Dwyer-Lindgren $L$, Lofgren $K T$, Rajaratnam JK, Marcus JR, Levin-Rector A, Levitz C, Lopez AD, Murray CJL. Age-specific and sex-specific mortality in 187 countries, 1970-2010: a systematic analysis for the Global Burden of Disease Study 2010. The Lancet. 2012 Dec 13; 380: 2071-2094.

3. UNICEF. Levels and trends in child mortality report, 2013 - estimates developed by the UN Interagency Group for Child Mortality Estimation. http://www.childinfo.org/files/ Child_Mortality_Report_2013.pdf. Accessed November 20, 2013.

4. UNICEF. 2013 Statistical snapshot. Child mortality. http://www.childinfo.org/files/ Child_Mortality_Stat_Snapshot_eversion_Sep_17.pdf. Accessed November 20, 2013.
5. Black RE, Cousens S, Johnson HL, et al. Global, regional, and national causes of child mortality in 2008: a systematic analysis. Lancet. 2010 Jun 5;375(9730):1969-87. doi: 10.1016/ S0140-6736(10)60549-1. Epub 2010 May 11.

6. Denno DM. Global Child Health. Pediatr Rev. 32;e25-e38, 2011.

7. United nations - The Millennium Development Goals Report, 2013. http://www.un.org/ millenniumgoals/pdf/report-2013/mdg-report2013-english.pdf. Accessed November 20, 2013.

8. World Health Organization - Children: reducing mortality. http://www. who.int/mediacentre/ factsheets/fs178/en/. Accessed November 20, 2013.

9. March of Dimes, et al., Born Too Soon: The Global Action Report on Preterm Birth, edited by Christopher P. Howson, Mary V. Kinney and Joy E. Lawn, World Health Organization, Geneva, 2012.

10. United Nations. Keeping the promise - United to achieve the millennium development goals. http://www.un.org/en/mdg/summit2010/pdf/ ZeroDraftOutcomeDocument_31May2010rev2.pdf. Accessed November 20, 2013 